\title{
Multi-purpose detector at the JINR NICA collider in the landscape of heavy-ion projects ${ }^{\star}$
}

\author{
Marek Gazdzicki ${ }^{1,2, a}$ \\ 1 Goethe-Universität Frankfurt am Main, Frankfurt, Germany \\ 2 Jan Kochanowski University, Kielce, Poland
}

\author{
Received: 18 December 2015 \\ Published online: 12 August 2016 \\ (C) The Author(s) 2016. This article is published with open access at Springerlink.com \\ Communicated by D. Blaschke
}

\begin{abstract}
The role of the multi-purpose detector at the JINR NICA collider in the landscape of heavy-ion projects is briefly discussed.
\end{abstract}

The multi-purpose detector (MPD) at the JINR NICA collider [1] is the third-generation experiment planned to study nucleus-nucleus collisions at energies of the CERN SPS. Heavy-ion physics in this energy range is very interesting for experimentalists and theorists mainly due to two reasons.

i) First, the NA49 experiment $[2,3]$ at the CERN SPS discovered a rapid change of the energy dependence of hadron production properties in central $\mathrm{Pb}+\mathrm{Pb}$ collisions which starts at about $p_{l a b}=30 \mathrm{AGeV} / \mathrm{c}$ $\left(\sqrt{s_{N N}} \approx 8 \mathrm{GeV}\right)$. This result, predicted as the signal of the onset of deconfinement (the phase transition to a quark-gluon plasma) at the low CERN SPS energies [4], is now confirmed by measurements at the BNL RHIC and CERN LHC, for a recent review see ref. [5]. Clearly, the NA49 study should be followed by systematic and precision measurements.

ii) Second, the critical point of strongly interacting matter may be located in the phase diagram region accessible in heavy-ion collisions at energies larger than the energy of the onset of deconfinement $\left(\sqrt{s_{N N}} \approx\right.$ $8 \mathrm{GeV}$ ), for a recent review see ref. [6]. Thus, measurements in this domain may lead to a discovery of the critical point.

There are three generations of experiments studying nucleus-nucleus collisions in the transition energy range:

i) NA49 [7] at the CERN SPS. The experiment performed the pilot study of the energy dependence of hadron production properties in central $\mathrm{Pb}+\mathrm{Pb}$ collisions, which resulted in the discovery of the rapid

\footnotetext{
* Contribution to the Topical Issue "Exploring strongly interacting matter at high densities - NICA White Paper" edited by David Blaschke et al.

a e-mail: marek.gazdzicki@cern.ch
}

changes of hadron production properties at the low SPS energies [2,3]. The detector was designed for the study of hadron production at the top SPS energy and not optimized for the performed energy scan. Data were registered in the period 1999-2002.

ii) NA61/SHINE [8] at the CERN SPS as well as STAR [9] and PHENIX [10] at the BNL RHIC. The second-generation experiments have performed measurements with the beams and detectors originally designed for the top SPS and RHIC energies and subsequently upgraded for measurements at the lower collision energies. The first phase of measurements is completed at RHIC [11] and it will be completed at SPS in 2017 [12]. The second phase is planned at both facilities $[13,14]$. The main results relevant for the MPD programme are: a confirmation of the NA49 discovery by STAR [15] and an observation of rapid changes of hadron production properties in $\mathrm{p}+\mathrm{p}$ interactions at the low SPS energies by NA61/SHINE [12]. Note that both results are still preliminary.

iii) MPD at the JINR NICA [1]. The third-generation experiment is planned to be performed using the dedicated accelerator and detector. The collision energy range will be $\sqrt{s_{N N}} \approx 4-11 \mathrm{GeV}$ and thus it covers the energy at which NA49 and NA61/SHINE report the evidence for the transition. The primary physics goal is to measure the collision-energy and system-size dependence of fluctuations and correlations with the aim to study properties of the transition region.

Note that the maximum collision energy of two other heavy-ion facilities under preparation, CBM at the FAIR SIS-100(300) [16] and the heavy-ion experiment at J-PARC [17], will be about $\sqrt{s_{N N}} \approx 5(8) \mathrm{GeV}$, i.e. below(at) the energy of the onset of deconfinement, $\approx 8 \mathrm{GeV}$. Thus, MPD is the only third-generation project covering the transition domain. 
The above provokes a number of comments concerning MPD at the JINR NICA and, in general, possible other future third-generation projects.

i) Planning and construction of the NICA project starts well before the results of the second-generation experiments are completed. In particular, it may happen [6] that the critical point will be discovered close to the top SPS energy, $\sqrt{s_{N N}} \approx 17 \mathrm{GeV}$, i.e. above the maximum energy of the JINR NICA. It would be important to consider a possibility of future upgrades to higher energies.

ii) The performance of the second-generation experiments is limited by the use of the facilities which were originally designed for other studies. This, in particular, negatively impacts measurements of event-byevent fluctuation and correlation. The third-generation projects should be designed in order to overcome these limitations. This implies:

- measurements of charged particles in the full acceptance,

- measurements of proton and neutron spectators from both colliding nuclei,

- an almost unique particle identification in a large acceptance,

- scanning in two-dimensional planes, (system-size)(energy) and (centrality)-(energy).

Open Access This is an open access article distributed under the terms of the Creative Commons Attribution License (http://creativecommons.org/licenses/by/4.0), which permits unrestricted use, distribution, and reproduction in any medium, provided the original work is properly cited.

\section{References}

1. A. Sorin, V. Kekelidze, A. Kovalenko, R. Lednicky, V. Matveev, I. Meshkov, G. Trubnikov, PoS (CPOD 2014) 042 (2015).

2. NA49 Collaboration (S.V. Afanasiev et al.), Phys. Rev. C 66, 054902 (2002) nucl-ex/0205002.

3. NA49 Collaboration (C. Alt et al.), Phys. Rev. C 77, 024903 (2008) arXiv:0710.0118 [nucl-ex].

4. M. Gazdzicki, M.I. Gorenstein, Acta Phys. Pol. B 30, 2705 (1999) hep-ph/9803462.

5. M. Gazdzicki, M.I. Gorenstein, P. Seyboth, Int. J. Mod. Phys. E 23, 1430008 (2014) arXiv:1404.3567 [nucl-ex].

6. M. Gazdzicki, P. Seyboth, arXiv:1506.08141 [nucl-ex].

7. NA49 Collaboration (S. Afanasev et al.), Nucl. Instrum. Methods A 430, 210 (1999).

8. NA61 Collaboration (N. Abgrall et al.), JINST 9, P06005 (2014) arXiv:1401.4699 [physics.ins-det].

9. STAR Collaboration (K.H. Ackermann et al.), Nucl. Instrum. Methods A 499, 624 (2003).

10. PHENIX Collaboration (K. Adcox et al.), Nucl. Instrum. Methods A 499, 469 (2003).

11. STAR Collaboration (D. McDonald), EPJ Web of Conferences 95, 01009 (2015).

12. NA61/SHINE Collaboration (A. Aduszkiwicz et al.), CERN-SPSC-2015-036; SPSC-SR-171, http://cds . cern. $\mathrm{ch} /$ record/2059310? $1 \mathrm{n}=$ en.

13. NA61/SHINE Collaboration (A. Aduszkiwicz et al.), CERN-SPSC-2015-038; SPSC-P-330-ADD-8, http:// cds. cern. ch/record/2059811? ln=en.

14. G. Odyniec, EPJ Web of Conferences 95, 03027 (2015).

15. D. Cebra for the STAR Collaboration, CPOD 2014, Bielefeld, November 2014, http://www.physik.unibielefeld.de/ cpod2014/CPOD2014_Cebra_ver2.pptx.

16. P. Senger, JPS Conf. Proc. 8, 022001 (2015).

17. H. Sako et al., Nucl. Phys. A 931, 1158 (2014). 